\title{
Management of Severe Acute Pancreatitis: An Update
}

\author{
Nina Gliem Christoph Ammer-Herrmenau Volker Ellenrieder
}

Albrecht Neesse

Gastroenterology and Gastrointestinal Oncology, University Medical Center Goettingen, Goettingen, Germany

\section{Key Points}

- Persistent organ failure ( $>48 \mathrm{~h}$ ) is the strongest predictor of poor prognosis.

- Early targeted fluid therapy within the first $48 \mathrm{~h}$ is critical to improve the outcome.

- Thoracic epidural analgesia might have prognostically beneficial effects in select patients.

- Enteral feeding should be started early during severe AP.

- Endoscopic ultrasound-guided drainage (double plastic pigtails or lumen-apposing metal stent [LAMS]) is the first choice for drainage of necrotic collections.

\section{Keywords}

Pancreatitis · Fluid therapy · Antibiotics · Organ failure · Walled-off necrosis

\section{Abstract \\ Background: Severe acute pancreatitis (AP) continues to be a serious gastrointestinal disease with relevant morbidity and mortality. Summary: Successful clinical management requires close interdisciplinary cooperation and coordina- tion from experienced gastroenterologists, intensive care physicians, surgeons, and radiologists. While the early phase of the disease is characterized by intensive care aspects that focus primarily on treatment of organ failure, later complica- tions are characterized especially by (infected) necrotic col- lections. Here, we discuss current clinical standards and de- velopments for conservative and interventional manage- ment of patients with severe AP. Key messages: Early targeted fluid therapy within the first $48 \mathrm{~h}$ is critical to im- prove the outcome of severe AP. Thoracic epidural analgesia}

may have prognostically beneficial effects due to suspected anti-inflammatory effects and increased perfusion of splanchnic vessels. Enteral feeding should be started early during severe AP. Persistent organ failure ( $>48 \mathrm{~h}$ ) is the strongest predictor of poor prognosis, and local complications such as infected walled-off necrosis should be primarily treated by minimally invasive endoscopic step-up approaches that are usually superior to surgical therapy options.

$$
\begin{aligned}
& \text { (c) } 2020 \text { The Author(s) } \\
& \text { Published by S. Karger AG, Basel }
\end{aligned}
$$

\section{Symptoms and Diagnosis}

The diagnosis of acute pancreatitis (AP) is based on elevated serum lipase levels (at least 3 times the upper limit of normal) and abdominal pain, which may radiate to the back. Despite common clinical practice, routine CT is not indicated and not recommended by national and international guidelines for diagnosis in patients with a

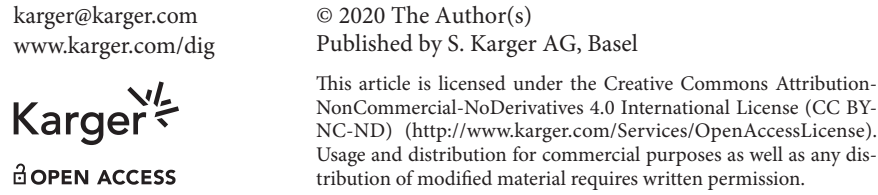

Dr. Albrecht Neesse

Department of Gastroenterology and Gastrointestinal Oncology University Medical Centre Goettingen

Robert-Koch-Strasse 40, DE-37075 Goettingen (Germany) albrecht.neesse@med.uni-goettingen.de 
Table 1. Harmless Acute Pancreatitis Score

No signs of peritonitis (rebound tenderness, guarding) Normal hematocrit level Serum Cr level $\leq 2 \mathrm{mg} / \mathrm{dL}$

typical clinical presentation [1]. In addition to routine laboratory testing (blood count, lipase, C-reactive protein, glucose, calcium, and liver and cholestasis enzymes), transabdominal ultrasonography belongs to basic examinations and can often help identify the triggering cause of AP, especially in patients with gallstones, acute cholecystitis, and intra- or extrahepatic cholestasis. A clear indication for early CT exists only in cases of diagnostic uncertainty, suspicion of organ perforation, or acute intra-abdominal bleeding (e.g., due to an arrosion of mesenteric vessels).

\section{Early Assessment of Severity}

In about $80 \%$ of cases, AP occurs as a mild edematous form, and patients can be discharged after only few days. However, in approximately $20 \%$ of patients, a severe or complicated course of pancreatitis develops, which is characterized by early or delayed systemic and local complications. In severe AP, mortality can reach up to $50 \%$, which is in contrast to a total mortality of $2-5 \%$ for all forms of AP. Early ( $<24 \mathrm{~h})$ assessment of the severity of pancreatitis is still a challenge in the clinic and has not yet been satisfactorily addressed. There are a variety of clinical and intensive care scores that can help retrospectively determine the severity and mortality of AP (e.g., Ranson score, Balthazar score, SOFA score, APACHE II score, and Marshall score). However, none of these scores is widely applied in clinical routine outside of clinical trials. The Harmless Acute Pancreatitis Score (HAPS) is a score developed in Germany that can predict mild forms of pancreatitis at the time of admission with high accuracy [2] (Table 1).

To determine severe AP, the Bedside Index for Severity in Acute Pancreatitis (BISAP) score can be used [3] (Table 2). A BISAP score $\geq 3$ can predict a severe course of AP with a sensitivity of $83 \%$. More recently, the Pancreatitis Activity Scoring System (PASS) was introduced that considers factors such as organ failure, pain, intolerance to solid diet, systemic inflammatory response syndrome, and morphine equivalent dose by relative weight [4]. In addition to these scoring systems, differ-
Table 2. Bedside Index for Severity in Acute Pancreatitis (BISAP) score

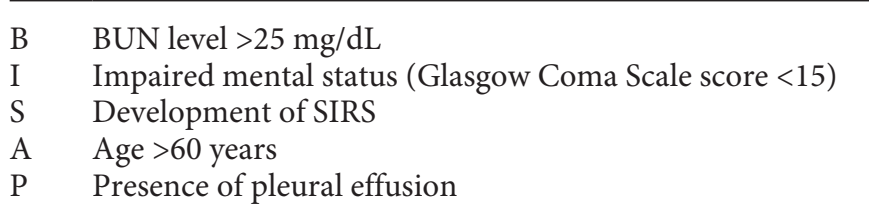

SIRS, systemic inflammatory response syndrome.

ent ancillary clinical and laboratory criteria may indicate a mild or a severe AP on admission, although inconsistent cutoff values often complicate matters. Nonetheless, a high C-reactive protein (>150 mg/L), hypocalcemia, increased hematocrit, or hyperglycemia more likely indicates a severe course of pancreatitis. Similarly, a short period $(<18 \mathrm{~h})$ from the onset of pain to presentation in the emergency department is associated with a severe course [5].

\section{Moderately Severe and Severe AP}

A moderately severe or a severe AP is characterized by the presence of a transient $(<48 \mathrm{~h})$ or persistent organ failure $(>48 \mathrm{~h}$ ) or local complications according to the revised Atlanta classification [6]. Organ failure often manifests with respiratory, renal, or cardiovascular failure. Usually, admission to an intermediate care or intensive care unit is necessary. However, persistent organ failure $(>48 \mathrm{~h})$ is associated with a significantly increased mortality of up to $50 \%$. A recent published post hoc analysis of the Dutch Pancreatitis Study Group of 639 patients showed no association between the time of onset (in the first week or the second week) or the duration of persistent organ failure ( $<1$ week to $>3$ weeks) and mortality in patients with AP [7]. The most important cause for early mortality is a fulminant organ failure with a systemic inflammatory response syndrome. The early phase of AP is not caused by bacterial sepsis. Rather, it is triggered by a sterile inflammatory response caused by intracellular activation of proteases, which induce autodigestion of the pancreas, mainly the acinar cells. As a result, macrophages, lymphocytes, and neutrophilic granulocytes are rapidly activated and inflammatory mediators such as IL- 6 and IL- 8 are subsequently released. This increases the systemic inflammatory response and organ failure, especially through capillary leakage [8]. 
Furthermore, the presence of paralytic ileus or abdominal compartment syndrome should also be considered (measurement of intra-abdominal pressure via a bladder catheter) so that therapy can be initiated timely.

\section{Treatment of Severe Complicated Pancreatitis}

\section{Fluid Resuscitation}

Numerous studies have investigated the type and amount of intravenous fluid resuscitation in severe AP. However, there are currently no standardized recommendations, since the amount of volume depletion due to extracellular leakage may vary widely individually, and also the tolerated volume and the infusion rate depend on comorbidities such as heart failure, renal failure, and pulmonary diseases. Fluid resuscitation between 150 and 250 $\mathrm{mL} / \mathrm{h}$ in the first $24-48 \mathrm{~h}$ seems reasonable considering the current literature. Key points regarding fluid resuscitation are given as follows:

1. Adequate intravenous fluid resuscitation should be performed during the first $24-48 \mathrm{~h}$; delayed or insufficient fluids worsen the prognosis: in particular, mortality is increased $[9,10]$.

2. High-volume fluid therapy $(1,000 \mathrm{~mL} / \mathrm{h})$ may worsen the prognosis and should be avoided [11].

3. The best fluid to use is Ringer's lactate. The infusion rate should be continuously monitored within the first $24 \mathrm{~h}$ and adjusted based on urine excretion (target: $0.5-1 \mathrm{~mL} / \mathrm{kg} / \mathrm{h}$ ) and the vital parameters.

There is evidence that central venous pressure is not a reliable parameter for the assessment of volume responsiveness in AP, as central venous pressure is heavily affected by intra-abdominal pressure, which is often greatly elevated in AP [12]. Transpulmonary thermodilution-based hemodynamic monitoring or stroke volume variation of arterial blood pressure is currently investigated in studies (e.g., EAGLE study, NCT03082469) to target volume therapy.

\section{Analgesia, Antibiotics, and Nutrition}

Sufficient intravenous analgesia is always indicated in severe AP and is usually combined with opioids (e.g., pethidine) and nonopioids (e.g., metamizole). If intravenous analgesia fails to provide sufficient relief or enhances bowel paralysis, the use of thoracic epidural analgesia may be considered, as this was associated with improved survival in a multicenter retrospective trial [13]. However, the potential benefits have to be confirmed in large randomized trials, and a recent randomized pilot study
( $n=16$ patients) showed a beneficial trend but no significant improvement in organ dysfunction or mortality upon thoracic epidural analgesia [14].

The routine use of antibiotics in the early course of severe AP is currently not recommended due to a lack of clear evidence [15]. A recently published retrospective multicenter cohort study of more than 800 patients showed that antibiotic prophylaxis in patients with severe AP may lead to the development of invasive candidiasis of the pancreas [16]. Further studies must clarify whether antimicrobial prophylaxis may be beneficial in certain subgroups of severe AP. If cholangitis or other local infections, for example, infected walled-off necrosis (WON), is suspected, intravenous antibiotics are recommended.

With regard to nutrition, a liberal management has been established in recent years. If possible, stepwise oral feeding should be encouraged within the first few days after admission [17]. A longer period of fasting should be avoided. In severely ill intensive care patients without gastrointestinal paralysis, a nasogastric or nasoduodenal feeding tube should be inserted carefully, as the outcome is significantly improved compared to that using total parenteral nutrition [18]. In patients with oral feeding intolerance, small-volume enteral feeding in combination with parenteral nutrition can be considered.

\section{Role of Endoscopy in the Treatment of Severe Necrotizing Pancreatitis}

\section{Drainage of Necrotic Collections}

Severe pancreatitis often develops acute (necrotic) fluid collections in the abdomen, which can encapsulate with increasing time (from about 4 weeks) and demarcate as WON.

About $40 \%$ of patients with necrotizing pancreatitis develop organ failure [8]. Necrotic collections can remain sterile and, if no symptoms occur, can be managed conservatively, as spontaneous resolution is possible. In the case of suspected or confirmed infection, gastric outlet or biliary tract compression, or even sepsis, interventional drainage is indicated, sometimes even before complete formation of WON if fulminant sepsis develops.

In most cases, an endoscopic ultrasound-guided transduodenal or transgastric drainage with placement of a plastic or a lumen-apposing metal stent (LAMS) can be performed and is superior to open necrosectomy and minimally invasive surgery [19-22]. Whether multiple plastic pigtails or LAMSs are superior to drain necrotic collections has not been finally answered: in some studies, 
Fig. 1. Transgastric LAMS placed in the small curvature with fresh drainage of infected necrosis.

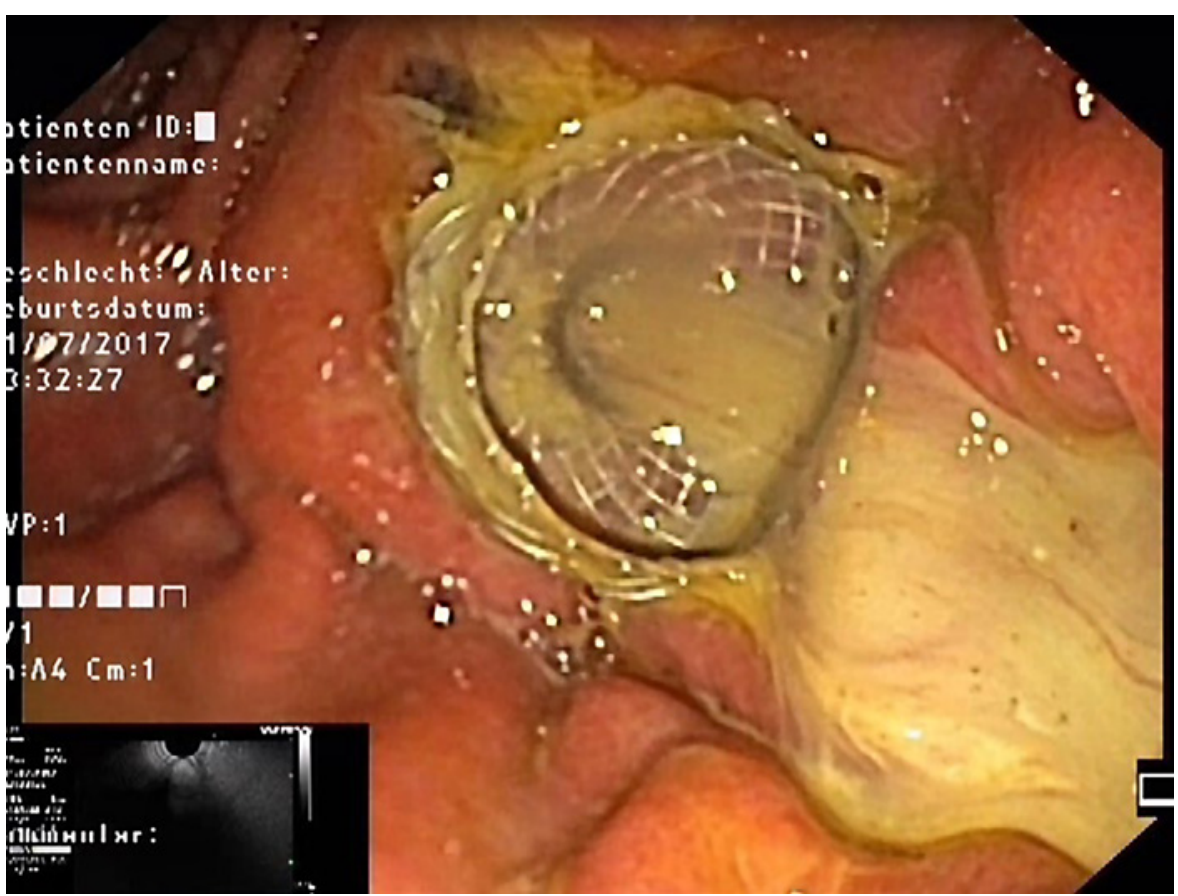

the advantages of LAMSs prevailed [23]; however, the only randomized controlled trial comparing LAMSs versus plastic pigtails did not show relevant differences in efficacy and clinical outcome [24]. In general, LAMSs provide a quick and relatively large access to the necrotic collection (usually 10-20 $\mathrm{mm}$ in diameter), thus allowing endoscopic necrosectomy with irrigation or insertion of a transgastric drainage (Fig. 1). According to current recommendations, LAMSs must be removed after approximately 4 weeks due to a potentially increased bleeding risk. In contrast, placement of plastic pigtails is endoscopically more demanding and time-consuming and does not allow necrosectomy. However, transgastric or transduodenal pigtails may remain in situ for several months. An initial LAMS placement followed by a plastic pigtail through the same access upon removal of the LAMS seems a feasible solution if the WON is incompletely resolved after 4 weeks. External drainage by ultrasound or $\mathrm{CT}$ guidance can be performed if endoscopic drainage is not feasible or available. Due to the significantly improved, minimally invasive endoscopic treatment options, surgical procedures for severe necrotic pancreatitis have decreased dramatically. Combining external and internal drainage, possibly also combined with minimally invasive video-assisted retroperitoneal debridement, allows for a step-up approach for individualized therapy of these seriously ill patients, significantly reducing the rate of major complications compared to the primary open surgical procedure [25]. A recently published randomized study showed that transluminal endoscopic intervention is associated with fewer complications and costs and an improved quality of life compared to minimally invasive video-assisted retroperitoneal debridement [21]. Nevertheless, a surgical procedure may still be required in individual cases and should always be discussed in an interdisciplinary manner.

\section{Urgent ERC in Patients with Severe Biliary \\ Pancreatitis}

According to the guidelines, urgent endoscopic retrograde cholangiography (ERC) is indicated in case of biliary pancreatitis with acute cholangitis [1]. However, whether an ERC should be performed within the first 24$72 \mathrm{~h}$ in the presence of biliary pancreatitis without cholangitis has not been conclusively answered. Different, partly very small meta-analyses had yielded inconsistent results, but the results suggested a potential benefit of an early ERC in severe biliary pancreatitis with cholestasis [26]. However, the data of the Dutch APEC trial (randomized, multicentric), presented only in an abstract form until now, suggest no benefit of an early ERC in severe AP without the presence of cholangitis [27]. However, future studies will have to address whether selection of patients using endoscopic ultrasound for detection of common bile duct stones in severe biliary pancreatitis results in a different outcome. 


\section{Statement of Ethics}

The authors have no ethical conflicts to disclose.

\section{Disclosure Statement}

The authors have no conflicts of interest to declare.

\section{Funding Sources}

The authors did not receive any funding.

\section{Author Contributions}

N.G. and A.N. wrote the initial version of the manuscript. C.A.M. and V.E. critically revised the manuscript and compiled figures and tables.

\section{References}

1 Working Group IAPAPAAPG. IAP/APA evidence-based guidelines for the management of acute pancreatitis. Pancreatology. 2013 Jul-Aug;13(4 Suppl 2):e1-5.

2 Lankisch PG, Weber-Dany B, Hebel K, Maisonneuve $\mathrm{P}$, Lowenfels $\mathrm{AB}$. The harmless acute pancreatitis score: a clinical algorithm for rapid initial stratification of nonsevere disease. Clin Gastroenterol Hepatol. 2009 Jun;7(6):702-607; quiz 607.

3 Singh VK, Wu BU, Bollen TL, Repas K, Maurer R, Johannes RS, et al. A prospective evaluation of the bedside index for severity in acute pancreatitis score in assessing mortality and intermediate markers of severity in acute pancreatitis. Am J Gastroenterol. 2009 Apr; 104(4):966-71.

4 Buxbaum J, Quezada M, Chong B, Gupta N, $\mathrm{Yu}$ CY, Lane $\mathrm{C}$, et al. The pancreatitis activity scoring system predicts clinical outcomes in acute pancreatitis: findings from a prospective cohort study. Am J Gastroenterol. 2018 May;113(5):755-64.

5 Brown A, Baillargeon JD, Hughes MD, Banks PA. Can fluid resuscitation prevent pancreatic necrosis in severe acute pancreatitis? Pancreatology. 2002;2(2):104-7.

6 Banks PA, Bollen TL, Dervenis C, Gooszen HG, Johnson CD, Sarr MG, et al. Classification of acute pancreatitis-2012: revision of the Atlanta classification and definitions by international consensus. Gut. 2013 Jan;62(1):102-11.

7 Schepers NJ, Bakker OJ, Besselink MG, Ahmed Ali U, Bollen TL, Gooszen HG, et al. Impact of characteristics of organ failure and infected necrosis on mortality in necrotising pancreatitis. Gut. 2019 Jun;68(6):1044-51.

8 Mayerle J, Sendler M, Hegyi E, Beyer G, Lerch MM, Sahin-Toth M. Genetics, cell biology, and pathophysiology of pancreatitis. Gastroenterology. 2019 May;156(7):1951-68 e1.

9 Gardner TB, Vege SS, Chari ST, Petersen BT, Topazian MD, Clain JE, et al. Faster rate of initial fluid resuscitation in severe acute pancreatitis diminishes in-hospital mortality. Pancreatology. 2009;9(6):770-6.

10 Warndorf MG, Kurtzman JT, Bartel MJ, Cox M, Mackenzie T, Robinson S, et al. Early fluid resuscitation reduces morbidity among patients with acute pancreatitis. Clin Gastroenterol Hepatol. 2011 Aug;9(8):705-9.
11 Mao EQ, Fei J, Peng YB, Huang J, Tang YQ, Zhang SD. Rapid hemodilution is associated with increased sepsis and mortality among patients with severe acute pancreatitis. Chin Med J. 2010 Jul;123(13):1639-44.

12 Huber W, Umgelter A, Reindl W, Franzen M, Schmidt C, von Delius S, et al. Volume assessment in patients with necrotizing pancreatitis: a comparison of intrathoracic blood volume index, central venous pressure, and hematocrit, and their correlation to cardiac index and extravascular lung water index. Crit Care Med. 2008 Aug;36(8):2348-54.

13 Jabaudon M, Belhadj-Tahar N, Rimmelé T, Joannes-Boyau O, Bulyez S, Lefrant JY, et al. Thoracic epidural analgesia and mortality in acute pancreatitis: a multicenter propensity analysis. Crit Care Med. 2018 Mar;46(3): e198-205.

14 Tyagi A, Gupta YR, Das S, Rai G, Gupta A Effect of segmental thoracic epidural block on pancreatitisinduced organ dysfunction: a preliminary study. Indian J Crit Care Med. 2019 Feb;23(2):89-4

15 Arlt A, Erhart W, Schafmayer C, Held HC, Hampe J. Antibiosis of necrotizing pancreatitis. Viszeralmedizin. 2014 Oct;30(5):318-24.

16 Horibe M, Sanui M, Sasaki M, Honda H, Ogura Y, Namiki S, et al. Impact of antimicrobial prophylaxis for severe acute pancreatitis on the development of invasive candidiasis: a large retrospective multicenter cohort study. Pancreas. 2019 Apr;48(4):537-43.

17 Bakker OJ, van Brunschot S, van Santvoort HC, Besselink MG, Bollen TL, Boermeester MA, et al. Early versus on-demand nasoenteric tube feeding in acute pancreatitis. N Engl J Med. 2014 Nov;371(21):1983-93.

18 Hsieh PH, Su HY, Lin CY, Kang YN, Chang CC. Infection rate among nutritional therapies for acute pancreatitis: a systematic review with network meta-analysis of randomized controlled trials. PLoS One. 2019;14(7): e0219151.
19 van Santvoort HC, Besselink MG, Bakker OJ, Hofker HS, Boermeester MA, Dejong CH, et al. A step-up approach or open necrosectomy for necrotizing pancreatitis. N Engl J Med. 2010 Apr;362(16):1491-502.

20 van Brunschot S, van Grinsven J, van Santvoort $\mathrm{HC}$, Bakker OJ, Besselink MG, Boermeester MA, et al. Endoscopic or surgical step-up approach for infected necrotising pancreatitis: a multicentre randomised trial. Lancet. 2018 Jan;391(10115):51-8.

21 Bang JY, Arnoletti JP, Holt BA, Sutton B, Hasan MK, Navaneethan U, et al. An endoscopic transluminal approach, compared with minimally invasive surgery, reduces complications and costs for patients with necrotizing pancreatitis. Gastroenterology. 2019 Mar;156(4):1027-e3.

22 Hollemans RA, Bakker OJ, Boermeester MA, Bollen TL, Bosscha K, Bruno MJ, et al. Superiority of step-up approach vs. open necrosectomy in long-term follow-up of patients with necrotizing pancreatitis. Gastroenterology. 2019 Mar;156(4):1016-26.

23 Bazerbachi F, Sawas T, Vargas EJ, Prokop LJ, Chari ST, Gleeson FC, et al. Metal stents versus plastic stents for the management of pancreatic walled-off necrosis: a systematic review and meta-analysis. Gastrointest Endosc. 2018 Jan;87(1):30-42 e15.

24 Bang JY, Navaneethan U, Hasan MK, Sutton B, Hawes R, Varadarajulu S. Non-superiority of lumen-apposing metal stents over plastic stents for drainage of walled-off necrosis in a randomised trial. Gut. 2019 Jul;68(7):1200-9.

25 Besselink MG. The 'step-up approach' to infected necrotizing pancreatitis: delay, drain, debride. Dig Liver Dis. 2011 Jun;43(6):421-2.

26 van Santvoort HC, Besselink MG, de Vries AC, Boermeester MA, Fischer K, Bollen TL, et al. Early endoscopic retrograde cholangiopancreatography in predicted severe acute biliary pancreatitis: a prospective multicenter study. Ann Surg. 2009 Jul;250(1):68-75.

27 Schepers NJ, Bakker OJ, Besselink MG, Bollen TL, Dijkgraaf MG, van Eijck CH, et al. Early biliary decompression versus conservative treatment in acute biliary pancreatitis (APEC trial): study protocol for a randomized controlled trial. Trials. 2016 Jan; 17:5. 